\title{
Ecuaciones de aditividad para la estimación de biomasa aérea de Pinus cembroides Zucc.
}

\section{Additive equations for estimating aboveground biomass of Pinus cembroides Zucc.}

\author{
Juan Carlos Cuevas Cruz"* y Martín Aquino Ramírez²
}

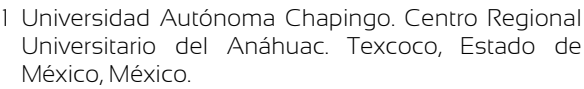

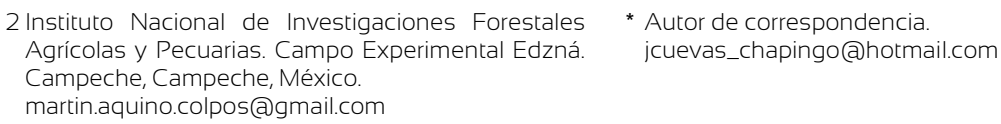

2 Instituto Nacional de Investigaciones Forestales Agrícolas y Pecuarias. Campo Experimental Edzná. Campeche, Campeche, México.

martin.aquino.colpos@gmail.com

* Autor de correspondencia. jcuevas_chapingo@hotmail.com

\section{RESUMEN}

Las ecuaciones alométricas son una herramienta importante para la estimación precisa de la biomasa aérea de los árboles y de sus componentes. En este documento se presentan ecuaciones de aditividad para estimar la distribución de la biomasa aérea total en árboles de Pinus cembroides Zucc. En octubre de 2017, un total de 21 árboles de distintas clases diamétricas $(5 \mathrm{~cm}$ a $40 \mathrm{~cm})$ fueron derribados y seccionados en tres componentes estructurales: fuste, ramas y ramillas con hojas, para obtener su biomasa. Se formularon dos sistemas de ecuaciones no lineales, en el que la aditividad se garantizó al establecer a la biomasa aérea total como la suma de las estimaciones de la biomasa de cada componente del árbol. Los sistemas de ecuaciones fueron ajustados simultáneamente con el Método Generalizado de Momentos (GMM, por sus siglas en inglés). El sistema que integró al diámetro normal y la altura total del árbol como variables predictoras explicó más del $92 \%$ de la variación observada en los datos de biomasa de los componentes y $98 \%$ de variabilidad de la biomasa aérea total. La proporción promedio de biomasa en el fuste, ramas y ramillas con hojas comprendió $43.2 \%, 41.2 \%$ y $15.5 \%$ de la biomasa aérea total del árbol, respectivamente. Las ecuaciones generadas en este estudio contribuirán a mejorar la precisión en la estimación de la biomasa e inventarios de carbono en los bosques de P. cembroides.

Palabras Clave: distribución de biomasa, Método Generalizado de Momentos, modelos no lineales, muestreo destructivo, pino piñonero, sistema de ecuaciones.

\section{ABSTRACT}

Allometric equations are an important tool for accurate estimation of aboveground biomass of trees and their components. This document presents additive equations for estimating aboveground biomass in Pinus cembroides Zucc trees. In October 2017, a total of 21 trees of different diameter classes $(5 \mathrm{~cm}$ to $40 \mathrm{~cm}$ ) were felled and sectioned into three structural components: stem, branches and twigs with leaves to obtain their biomass. Two systems of non-linear equations were developed, in which the additivity was guaranteed by establishing the total aboveground biomass $(\mathrm{Bt})$ as the sum of the biomass estimates of each component of the tree. The system that integrated the diameter at breast height and the total height of the tree as predictor variables explained more than $92 \%$ of the variation observed in the biomass data of the components and $98 \%$ of variability of the Bt. The average proportion of biomass in the stem, branches and twigs with leaves comprised $43.2 \%, 41.2 \%$ and $15.5 \%$ of the tree $\mathrm{Bt}$, respectively. The equations generated in this study will contribute to improve the precision in biomass estimation and carbon inventories in P. cembroides forests.

KEYWORDS: biomass distribution, Generalized Method of Moments, nonlinear models, destructive sampling, pinyon pine, system of equations. 


\section{INTRODUCCIÓN}

La estimación de biomasa de los árboles es determinante en los estudios de productividad, ciclo de nutrientes y cuantificación de las contribuciones al secuestro de carbono por parte de los ecosistemas forestales (Tesfaye, BravoOviedo, Bravo y Ruiz-Peinado, 2015; Meng, et al., 2017). Así mismo, el conocimiento de las cantidades de biomasa sirve como un indicador ecológico para la sostenibilidad y para caracterizar la estructura y función de los bosques (Chave et al., 2005). En los últimos años, se ha incrementado la necesidad de disponer de información más eficiente sobre la cantidad de almacenamiento de biomasa y de los flujos de carbono forestal en los diferentes ecosistemas terrestres, debido a su influencia para mitigar el cambio climático global.

Para estimar la biomasa aérea en árboles se han generado ecuaciones alométricas, las cuales se derivan de la aplicación de un muestreo destructivo y del uso de técnicas de regresión (Acosta-Mireles, Vargas-Hernández, Velázquez-Martínez y Etchevers-Barra, 2002; VargasLarreta et al., 2017; Quiñonez-Barraza et al., 2019). Las ecuaciones de biomasa se fundamentan en las relaciones alométricas empíricas de los atributos del árbol que son fácilmente medibles como el diámetro normal, la altura total y la densidad específica de la madera (Temesgen, Affleck, Poudel, Gray y Sessions, 2015; Eker, Poudel y Özçelik, 2017). A nivel local o del sitio, el uso de ecuaciones resulta la opción más práctica en tiempo y costo para evaluar la cantidad de biomasa aérea a partir de datos de inventario forestal (Riofrío, Herrero, Grijalva y Bravo, 2015; Daryaei y Sohrabi, 2016). Diferentes estudios señalan que las ecuaciones específicas por especie mejoran las estimaciones de biomasa que las ecuaciones generales, porque se toman en cuenta las condiciones ambientales, la densidad de la madera y la variabilidad genética en donde se desarrollaron los árboles; factores que influyen significativamente en las relaciones alométricas (Skovsgaard y Vanclay, 2013; Lin et al., 2017; Mensah, Veldtman y Seifert, 2017).
Al estimar la biomasa de más de un componente del árbol es importante considerar la propiedad de aditividad puesto que se toman en cuenta las correlaciones inherentes entre los componentes medidos y la biomasa total, mejorando la precisión en las estimaciones (Carvalho y Parresol, 2003; Dong, Zhang y Li, 2014). Con la aditividad se asegura que la suma de los valores estimados de las ecuaciones de los componentes estructurales sea igual al valor estimado obtenido de la ecuación de la biomasa aérea total (Bi, Turner y Lambert, 2004).

En México, se han realizado considerables esfuerzos para generar ecuaciones para estimar la biomasa arbórea en las distintas especies de coníferas y latifoliadas (Douterlungne, Herrera-Gorocica, Ferguson, Siddique y Soto-Pinto, 2013; Aquino-Ramírez, Velázquez-Martínez, Castellanos-Bolaños, De los Santos-Posadas y EtcheversBarra, 2015; Rojas-García, De Jong, Martínez-Zurimendí y Paz-Pellat, 2015; Quiñonez-Barraza et al., 2019). La mayoría de las ecuaciones del género Pinus son restrictivas a especies con fines maderables (Chávez-Aguilar et al., 2016; VargasLarreta et al., 2017). Sin embargo, no existen ecuaciones para las especies de pinos piñoneros a pesar de su amplia distribución y plasticidad ambiental. Por lo cual, la información que se tiene de la capacidad de almacenamiento de biomasa y carbono de los bosques piñoneros es escasa.

Los bosques de Pinus cembroides Zucc. son característicos de las zonas áridas y de distribución latitudinal muy amplia sobre el altiplano del norte y centro de México (Luna-Cavazos, Romero-Manzanares y GarcíaMoya, 2008). P. cembroides es considerada como la principal especie de pinos piñoneros del país, crece sobre laderas de montaña en suelos delgados y pedregosos con climas de tipo templado seco a templado subhúmedo, donde la época de sequía puede durar de siete a ocho meses (ConstanteGarcía, Villanueva-Díaz, Cenaro-Paredes, Cornejo-Oviedo y Valencia-Manzo, 2009). A pesar de su limitada capacidad productividad puede constituir una fuente complementaria de captura y almacenamiento de carbono (Pompa-García y 
Yerena-Yamallel, 2014), además suministra bienes a los pobladores locales como leña y madera de cortas dimensiones, resina, árboles de navidad, alimento e ingresos económicos por la venta de su semilla (piñón). Por su amplia adaptabilidad y resistencia a condiciones extremas ha sido utilizada para proyectos de reforestación y restauración ecológica (Ríos, De Hoogh y Návar, 2008). No obstante, las poblaciones de esta especie se ven amenazadas o en peligro de desaparecer por las actividades antropogénicas como el cambio de uso del suelo, el pastoreo, los incendios, la extracción ilegal de la madera y principalmente por la recolección excesiva de la semilla (Rosas-Chavoya, Granados-Sánchez, Granados-Victorino y Esparza-Govea, 2016). Por lo anterior, son necesarias acciones que promuevan la conservación y el manejo sustentable de $P$. cembroides. Una alternativa viable es su incorporación a los programas de pago de servicios ambientales como la captura de carbono, puesto que estos bosques pueden considerarse como una posible fuente con potencial de mitigación ante el cambio climático (PompaGarcía y Yerena-Yamallel, 2014), aunque los montos económicos por la venta de bonos de carbono serían mínimos, debido a su baja productividad. De manera que se requiere información detallada de la cantidad de los diferentes componentes estructurales del árbol y total para estimar con precisión los almacenes de carbono en los bosques de $P$. cembroides.

\section{OBJETIVOS}

Desarrollar un sistema de ecuaciones de aditividad para estimar la biomasa de los componentes estructurales por separado y la biomasa aérea total a nivel de árbol individual para Pinus cembroides Zucc. en el bosque de la comunidad agraria de Sombrerete, Cadereyta de Montes, Querétaro, México.

\section{MATERIALES Y MÉTODOS}

\section{Área de estudio}

El estudio se realizó en el mes de octubre de 2017, en una superficie de 8 ha de las 1333 ha de los bosques naturales de la Comunidad Agraria de Sombrerete, Municipio de Cadereyta de Montes, Querétaro, México, ubicada entre los $20^{\circ} 49^{\prime} 80^{\prime \prime}$ у $20^{\circ} 50^{\prime} 0.05^{\prime \prime} \mathrm{N}$; y los $99^{\circ} 39^{\prime} 88.0^{\prime \prime}$ у $99^{\circ} 40^{\prime}$ 13.0" O, a $2600 \mathrm{~m}$ snm. El clima es de tipo semiseco (BS1 kw) con lluvias en verano. La temperatura media anual se encuentra entre $12{ }^{\circ} \mathrm{C}$ y $18{ }^{\circ} \mathrm{C}$, mientras que la temperatura del mes más frío llega a ser de $-3{ }^{\circ} \mathrm{C}$ y la del mes más cálido de $22^{\circ} \mathrm{C}$, con una precipitación media anual de $600 \mathrm{~mm}$ (García, 2004). Los análisis de laboratorio realizados indicaron que el tipo de suelo predominante es el Phaeozem con una textura franco-arcillo-arenosa, $\mathrm{pH}$ de 7.3 y un contenido de materia orgánica de $5.04 \%$. El tipo de vegetación corresponde a un bosque de pino dominado por P. cembroides y asociados a este, Juniperus flaccida Schltdl., Yucca queretaroensis Piña, Agave lechuguilla Torr. y Opuntia spp.

\section{Obtención de muestras y medición de la biomasa}

El método utilizado para la medición de biomasa aérea fue similar al propuesto por Picard, Saint-Andre y Henry (2012), que consiste en cortar a cada árbol a ras de suelo y separarlo en componentes estructurales. Un total de 21 individuos fueron derribados de distintas categorías diamétricas (CD) $(5 \mathrm{~cm}$ a $40 \mathrm{~cm}$ ). La distribución de los árboles fue de dos individuos para las CD de $5 \mathrm{~cm}, 10 \mathrm{~cm}$ y $30 \mathrm{~cm}$ y tres individuos para la $C D$ de $15 \mathrm{~cm}$; mientras que en las CD de $20 \mathrm{~cm}$ y $25 \mathrm{~cm}$ fue de cinco individuos y un individuo para las CD de $35 \mathrm{~cm}$ y $40 \mathrm{~cm}$. Los árboles seleccionados para su derribo estaban libres de plagas y enfermedades y no mostraban algún daño físico o mecánico. Previo al derribo, el diámetro normal $(D n)$ fue medido con cinta diamétrica a una altura de $1.30 \mathrm{~m}$ desde el nivel del suelo, mientras que la altura total $(H)$ fue medida con cinta métrica posterior al derribo como lo sugieren Acosta-Mireles et al. (2002) y Carrillo-Anzures, AcostaMireles, Jiménez-Cruz, González-Molina y EtcheversBarra, (2016). Los componentes estructurales en que se dividió cada árbol fueron: fuste, ramas y ramillas con hojas (diámetro $<1 \mathrm{~cm}$ ).

$\mathrm{El}$ fuste con corteza fue seccionado en trozas de $1.0 \mathrm{~m}$ de longitud desde la base hasta la punta o hasta la primera 
bifurcación del árbol, se obtuvieron muestras de ramas y muestras de ramillas con hojas, para ello la copa se dividió en tres secciones transversales: baja, media y alta (Zhang, Wang, Wang y Quan, 2009). De acuerdo con el procedimiento sugerido por Acosta-Mireles et al. (2002), Aquino-Ramírez et al. (2015) y Carrillo-Anzures et al. (2016) todos los componentes estructurales se pesaron por separado directamente en campo para obtener los pesos frescos totales $(\mathrm{kg})$ con una báscula digital de $200 \mathrm{~kg}$ (TECNOCOR modelo IPEN) y una precisión de $\pm 0.5 \mathrm{~kg}$. Después, una rodaja ( $3 \mathrm{~cm}-5 \mathrm{~cm}$ de grosor) fue obtenida en cada troza del fuste y tres rodajas al azar para las ramas en cada sección de la copa. De igual manera, para las ramillas con hojas se obtuvieron tres muestras al azar de 0.5 $\mathrm{kg}$ por sección. Las rodajas y muestras se pesaron inmediatamente con una báscula electrónica de $10 \mathrm{~kg}$ (TECNOCOR modelo PEN-20) con una precisión de \pm 5 g y puestas en bolsas de papel debidamente identificadas; luego fueron transportadas al laboratorio y colocadas por $96 \mathrm{~h}$ en una estufa de secado de madera (Nyle modelo L500) a $75^{\circ} \mathrm{C}$ o hasta lograr un peso constante. Finalmente, el peso seco de cada componente del árbol fue calculado extrapolando la relación peso seco a peso fresco de las muestras. La biomasa aérea total $(\mathrm{Bt})$ de cada árbol correspondió a la suma total de los pesos secos $(\mathrm{kg})$ de cada componente estructural (fuste, ramas y ramillas con hojas).

\section{Ecuaciones de biomasa}

Los siguientes dos modelos alométricos ampliamente utilizados, fueron seleccionados para estimar la biomasa de los componentes de P. cembroides (Ec. 1 y Ec. 2). Estos modelos relacionan a la biomasa como una función del $D n$ y la $H$ o la combinación de ambas variables $\left(D n^{2} H\right)(B i$, Turner y Lambert, 2004; Zhao, Kane, Markewitz, Teskey y Clutter, 2015):

$$
\begin{aligned}
& y=\exp \left[\beta_{0}\right] \times\left(D n^{2} H\right)^{\beta_{1}}+\varepsilon \\
& y=\exp \left[\beta_{0}\right] \times D n^{\beta_{1}} \times H^{\beta_{2}}+\varepsilon
\end{aligned}
$$

donde:

$y=$ biomasa de cualquiera de los componentes estructurales del árbol ( $\mathrm{kg})$

$D n=$ diámetro normal $(\mathrm{cm})$

$H=$ altura total $(\mathrm{m})$

$\beta_{0}, \beta_{1}$ y $\beta_{2}=$ parámetros de regresión

$\varepsilon=$ error aleatorio

El primer sistema de ecuaciones de aditividad $\left(S_{1}\right)$ fue basado en la ecuación 1 y formulado de la siguiente forma:

$$
\begin{aligned}
& B_{f}=\exp \left[\beta_{10}\right] \times\left(D n^{2} H\right)^{\beta_{11}}+\varepsilon_{1} \\
& B_{r}=\exp \left[\beta_{20}\right] \times\left(D n^{2} H\right)^{\beta_{21}}+\varepsilon_{2} \\
& B_{r h}=\exp \left[\beta_{30}\right] \times\left(D n^{2} H\right)^{\beta_{31}}+\varepsilon_{3}
\end{aligned}
$$

Como consecuencia de la aditividad del sistema, la estimación de la $\mathrm{Bt}$ del $\mathrm{S}_{1}$ será el resultado de la sumatoria de la estimación de la biomasa de los tres componentes, expresada de la siguiente manera:

$$
B_{t}=\sum_{i=1}^{3} \exp \left[\beta_{i 0}\right] \times\left(D n^{2} H\right)^{\beta_{i 1}}+\varepsilon_{4}
$$

Mientras que el segundo sistema de ecuaciones de aditividad $\left(\mathrm{S}_{2}\right)$ se fundamentó en la ecuación 2 y se especificó de la siguiente forma:

$$
\begin{aligned}
& B_{f}=\exp \left[\beta_{10}\right] \times D n^{\beta_{11}} \times H^{\beta_{12}}+\varepsilon_{1} \\
& B_{r}=\exp \left[\beta_{20}\right] \times D n^{\beta_{21}} \times H^{\beta_{22}}+\varepsilon_{2} \\
& B_{r h}=\exp \left[\beta_{30}\right] \times D n^{\beta_{31}} \times H^{\beta_{32}}+\varepsilon_{3}
\end{aligned}
$$

Y la aditividad de la Bt fue expresado como:

$$
B_{t}=\sum_{i=1}^{3} \exp \left[\beta_{i 0}\right] \times D n^{\beta_{i 1}} \times H^{\beta_{i 2}}+\varepsilon_{4}
$$


donde:

$\beta_{f},=$ biomasa en fuste $(\mathrm{kg})$

$\beta_{r}$, = biomasa en ramas $(\mathrm{kg})$

$\beta_{r b}=$ biomasa en ramillas con hojas $(\mathrm{kg})$

$\beta_{t},=$ biomasa total $(\mathrm{kg})$ en $\mathrm{kg}$ respectivamente;

$\beta_{i j}=$ coeficientes de regresión

$\varepsilon_{1}-\varepsilon_{4}=$ términos del error

En los dos sistemas de ecuaciones los errores son aditivos y están inherentemente correlacionados, los cuales son expresados como un vector

$$
\varepsilon=\left[\varepsilon_{1}, \varepsilon_{2}, \varepsilon_{3}\right]^{\prime}
$$

con un valor esperado

$$
E(\varepsilon)=0
$$

y una matriz de varianza y covarianza

$$
E\left(\varepsilon \varepsilon^{\prime}\right)=\sum
$$

(Bi et al., 2004; Wang, Bi, Ximenes, Ramos y Li, 2017). La propiedad de aditividad se obtuvo en los dos sistemas de ecuaciones al expresar a la biomasa aérea total (Bt) como la suma de la biomasa de cada uno de los componentes estructurales del árbol $\left(B_{f}, B_{r}, B_{r b}\right)$ (Parresol, 2001).

\section{Ajuste y comparación}

Los dos sistemas propuestos fueron ajustados simultáneamente usando el método generalizado de momentos (GMM, por sus siglas en inglés) con el procedimiento PROC MODEL del programa SAS/ETS $®$ (SAS Institute Inc., 2004). Con este método se estiman parámetros en ecuaciones no lineales con términos de error aditivo más eficientes en condiciones heterocedásticas sin ninguna especificación de su naturaleza (Greene, 1999), especialmente en muestras pequeñas, donde el principal problema de estimación es la obtención de funciones de ponderación de los componentes de biomasa que describan con precisión el diseño de la matriz de varianza del error (Bi et al., 2004; Bi et al., 2015). Para evaluar y comparar las ecuaciones desarrolladas en cada sistema se obtuvieron cuatro estadísticos: el coeficiente de determinación ajustado $\left(\mathrm{R}^{2}{ }_{a j j}\right)$, la raíz de los cuadrados medios del error (RMSE), el sesgo promedio $(\bar{S})$ y el error relativo promedio porcentual $(\bar{E})$ (Chave et al., 2005; Zhao et al., 2015). El sistema más apropiado fue el que presentó en sus ecuaciones el ajuste más alto $\left(R^{2}\right.$ adj) con un valor de $\bar{S}_{\text {cercano a cero y los valores }}$ más bajos de RMSE y $\bar{E}$ (Ec. 11, Ec. 12, Ec. 13, Ec. 14). Estos estadísticos se calcularon como:

$$
R_{A d j}^{2}=1-\left[\frac{\sum_{j=1}^{n}\left(y_{j}-\hat{y}_{j}\right)^{2}(n-p)}{\sum_{j=1}^{n}\left(y_{j}-\bar{y}\right)^{2} /(n-1)}\right]
$$

$$
R M S E=\sqrt{\sum_{j=1}^{n}\left(\hat{y}_{j}-y_{j}\right)^{2} / n-p}
$$

$$
\bar{S}=\sum_{j=1}^{n}\left(y_{j}-\hat{y}_{j}\right) / n
$$

$$
\bar{E}(\%)=\frac{100}{n} \sum_{j=1}^{n} \frac{\hat{y}_{j}-y_{j}}{y_{j}}
$$

donde:

$y_{j}=$ biomasa total del árbol observada

$\hat{y}_{j}=$ biomasa total del árbol predicha

$\bar{y}=$ biomasa total del árbol media

$n=$ número de observaciones

$p=$ número de parámetros del modelo

\section{Resultados}

\section{Variación en la distribución de biomasa aérea}

Los árboles derribados de P. cembroides en el presente estudio registraron un intervalo de diámetros de $3 \mathrm{~cm}$ a 40 $\mathrm{cm}$ con una media de $20.2 \mathrm{~cm} \pm 8.6 \mathrm{~cm}$ y alturas de $1.2 \mathrm{~m}$ a $9.4 \mathrm{~m}$ con un valor medio de $6.9 \mathrm{~m} \pm 1.9 \mathrm{~m}$; mientras que la biomasa total fue de $2.8 \mathrm{~kg}$ por árbol a $700.1 \mathrm{~kg}$ por árbol con un valor promedio de $180.8 \mathrm{~kg} \pm 163.8 \mathrm{~kg}$. Por otra 
parte, la distribución de biomasa entre los componentes estructurales (fuste, ramas y ramillas con hojas) mostraron importante variación debido a los tamaños del árbol (Fig. 1). El fuste y las ramas fueron los principales componentes del árbol donde se acumuló la mayor cantidad de biomasa y la de menor cantidad fueron las ramillas con hojas. La proporción en cuanto a la cantidad de biomasa del fuste manifestó una amplitud de valores entre $26.1 \%$ y $68.7 \%$ con un promedio de $43.2 \%$. En cambio, la proporción de la cantidad de biomasa en ramas fue mayor que en el fuste, siendo de $18.8 \%$ en las categorías diamétricas de $10 \mathrm{~cm}$ y de $64.6 \%$ en las categorías diamétricas de $40 \mathrm{~cm}$, con una media de $41.2 \%$; algo similar sucedió con la proporción de la biomasa en las ramillas con hojas y se presentó una variación de $6.3 \%$ a $32.1 \%$, en las categorías diamétricas de $5 \mathrm{~cm}$ a $10 \mathrm{~cm}$ con un valor medio de $15.5 \%$.

\section{Evaluación de las ecuaciones de biomasa}

El valor estimado, error estándar y la significancia de cada parámetro para los dos sistemas de ecuaciones de aditividad $\left(\mathrm{S}_{1}\right.$ y $\left.\mathrm{S}_{2}\right)$ son presentados en la tabla 1. Todos los parámetros fueron significativamente diferentes de cero $(\mathrm{p}<0.001)$. Los coeficientes de $\mathrm{R}^{2}$ adj obtenidos en las ecuaciones de distribución de biomasa se encontraron en un intervalo de $0.85 \%-0.97 \%$ y para las ecuaciones de biomasa aérea total fueron superiores a $0.97 \%$ (Tabla 1). La ecuación para predecir la biomasa de ramillas con hojas, que incluyó al $D n^{2} H$ como variable predictora fue la que mostró el valor más bajo de $R^{2}$ adj $(0.85)$ y valores altos de $\bar{S}$ y $\bar{E}$.

Los resultados de bondad de ajuste indicaron los mejores estadísticos en las ecuaciones del $\mathrm{S}_{2}$ que las obtenidas del $\mathrm{S}_{1}$. Las ecuaciones del $\mathrm{S}_{2}$ explicaron la mayor variabilidad de los datos observados con coeficientes de $\mathrm{R}^{2}$ adj superiores a 0.92 , así mismo, valores bajos de RMSE $(<25 \mathrm{~kg}), \bar{S}(<0.01 \mathrm{~kg})$ y $\bar{E}(<19 \%)$. Por lo tanto, el $\mathrm{S}_{2}$ fue seleccionado para la estimación de la biomasa de los componentes estructurales y biomasa aérea total de $P$. cembroides. Las curvas de predicción expresaron excelente comportamiento con los datos observados de biomasa (Fig. 2). Por otro lado, el diagnóstico gráfico de los residuales contra valores predichos en todas las ecuaciones no mostró tendencias visibles que indiquen heterocedasticidad (Fig. 3).

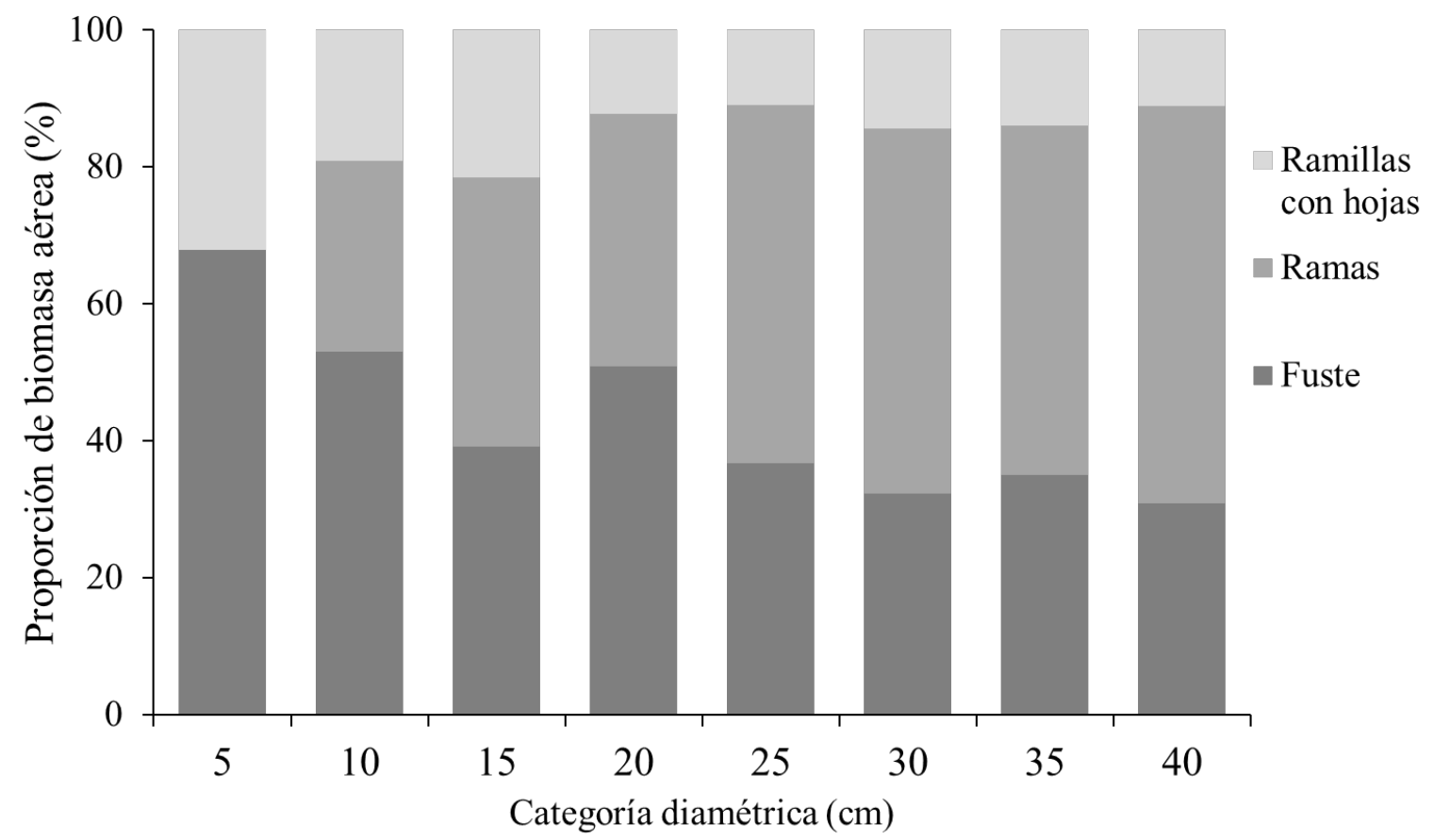

FIGURA 1. Proporción relativa de la biomasa aérea (\%) distribuida en el fuste, las ramas y las ramillas con hojas para las diferentes categorías diamétricas en Pinus cembroides. 
TABLA 1. Parámetros estimados y estadísticos de bondad de ajuste de las ecuaciones de aditividad para estimar la biomasa de los componentes estructurales y la biomasa aérea total de Pinus cembroides.

\begin{tabular}{|c|c|c|c|c|c|c|c|c|c|c|}
\hline $\begin{array}{l}\text { Sistema de } \\
\text { ecuaciones }\end{array}$ & $\begin{array}{c}\text { Componente } \\
\text { del árbol }\end{array}$ & Parámetro & Estimador & $\begin{array}{c}\text { Error } \\
\text { estándar }\end{array}$ & Valor de $t$ & $\operatorname{Pr}>|t|$ & RMSE (kg) & $\bar{S}_{(k g)}$ & $\bar{E}$ (\%) & $R_{A d j}^{2}$ \\
\hline \multirow{7}{*}{$S_{1}$} & \multirow{2}{*}{ Fuste } & $\beta_{10}$ & 2.766848 & 0.2151 & 12.86 & & \multirow{2}{*}{9.4955} & \multirow{2}{*}{0.5266} & \multirow{2}{*}{1.72} & \multirow{2}{*}{0.9641} \\
\hline & & $\beta_{11}$ & 0.848754 & 0.0233 & 36.43 & $<0.0001$ & & & & \\
\hline & \multirow{2}{*}{ Ramas } & $\beta_{20}$ & 4.182507 & 0.2549 & 16.41 & & \multirow{2}{*}{23.8367} & \multirow{2}{*}{-0.0715} & \multirow{2}{*}{30.27} & \multirow{2}{*}{0.9408} \\
\hline & & $\beta_{21}$ & 1.0513449 & 0.0276 & 38.05 & $<0.0001$ & & & & \\
\hline & \multirow{2}{*}{$\begin{array}{c}\text { Ramillas con } \\
\text { hojas }\end{array}$} & $\beta_{30}$ & 2.838132 & 0.3376 & 8.41 & $<0.0001$ & \multirow{2}{*}{7.1995} & \multirow{2}{*}{-0.4596} & \multirow{2}{*}{21.41} & \multirow{2}{*}{0.8597} \\
\hline & & $\beta_{31}$ & 0.738552 & 0.0357 & 20.71 & $<0.0001$ & & & & \\
\hline & \multirow[t]{2}{*}{ Total } & & & & & & \multirow[t]{2}{*}{25.3352} & -0.0035 & 5.00 & 0.9761 \\
\hline \multirow{10}{*}{$\mathrm{S}_{2}$} & & $\beta_{10}$ & -2.007449 & 0.3337 & 5.68 & $<0.0001$ & & \multirow{3}{*}{-0.0001} & \multirow{3}{*}{4.75} & \multirow{3}{*}{0.9704} \\
\hline & \multirow[t]{2}{*}{ Fuste } & $\beta_{11}$ & 1.776093 & 0.1028 & 17.28 & $<0.0001$ & \multirow[t]{2}{*}{8.6137} & & & \\
\hline & & $\beta_{13}$ & 0.364976 & 0.2675 & 1.36 & $<0.0001$ & & & & \\
\hline & \multirow{3}{*}{ Ramas } & $\beta_{20}$ & -5.193296 & 0.4592 & 11.31 & $<0.0001$ & \multirow{3}{*}{22.4340} & \multirow{3}{*}{0.0103} & \multirow{3}{*}{16.58} & \multirow{3}{*}{0.9476} \\
\hline & & $\beta_{21}$ & 2.36942 & 0.1743 & 13.59 & $<0.0001$ & & & & \\
\hline & & $\beta_{23}$ & 1.11539 & 0.4266 & 2.61 & 0.0176 & & & & \\
\hline & \multirow{3}{*}{$\begin{array}{c}\text { Ramillas con } \\
\text { hojas }\end{array}$} & $\beta_{30}$ & 2.07281 & 0.4878 & 4.25 & 0.0005 & \multirow{3}{*}{5.1074} & \multirow{3}{*}{0.0005} & & \\
\hline & & $\beta_{31}$ & 2.463058 & 0.1935 & 12.73 & $<0.0001$ & & & 19.12 & 0.9294 \\
\hline & & $\beta_{33}$ & -1.17492 & 0.4276 & -2.75 & 0.0132 & & & & \\
\hline & Total & & & & & & 23.0285 & 0.0116 & 5.76 & 0.9802 \\
\hline
\end{tabular}

SI=sistema de ecuaciones de aditividad basado en el modelo $y=\exp \left[\beta_{0}\right] \times\left(D n^{2} H\right)^{\beta_{1}}+\varepsilon ; \mathrm{S}_{2}=$ sistema de ecuaciones de aditividad basado en el modelo $y=\exp \left[\beta_{0}\right] \times D n^{\beta_{1}} \times H^{\beta_{2}}+\varepsilon$;

RMSE=raíz de los cuadrados medios del error; $\bar{S}$ = sesgo promedio; $\bar{E}$ = error relativo; $R_{A d j}^{2}=$ coeficiente de determinación ajustado 


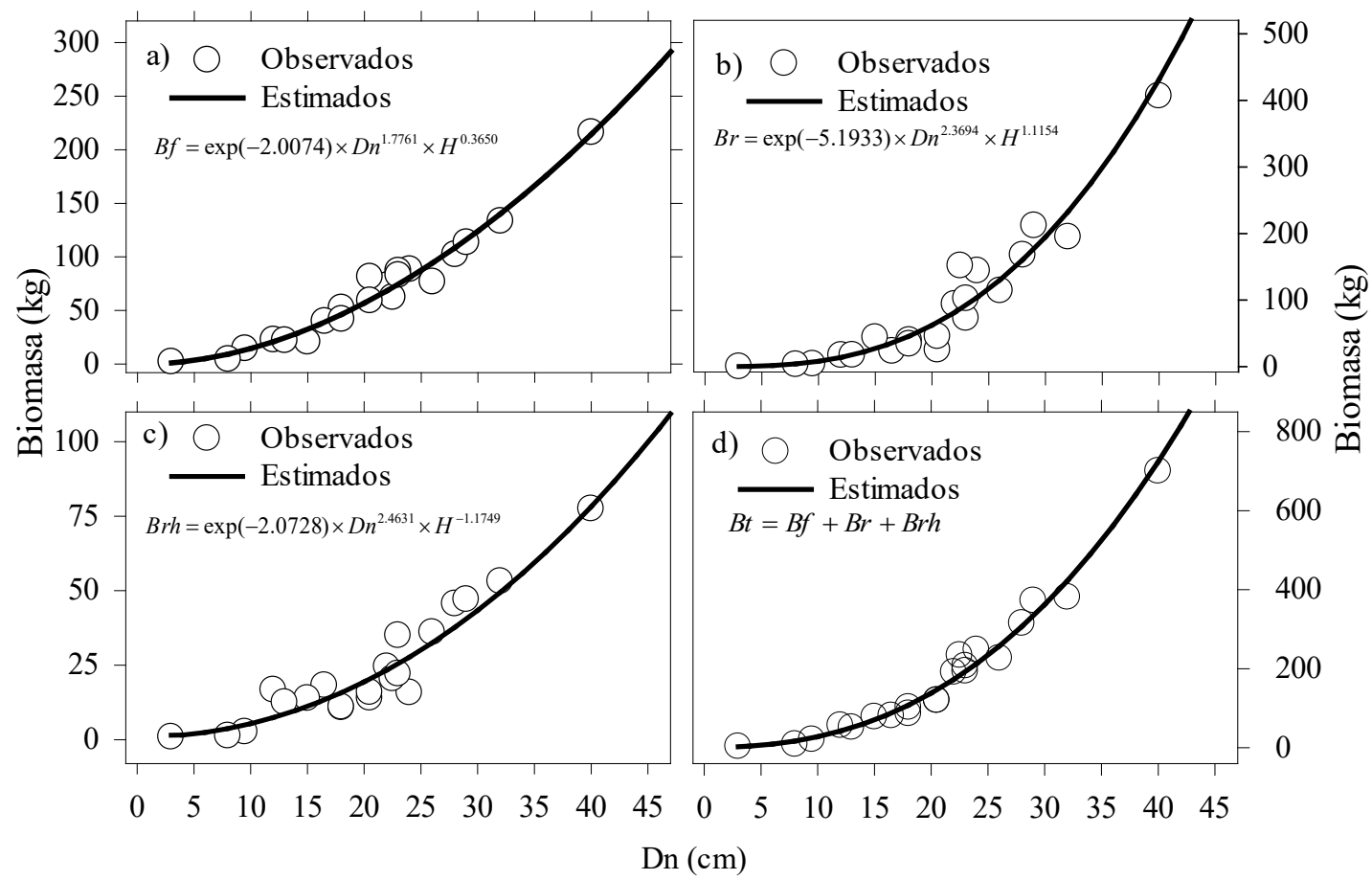

FIGURA 2. Comparación entre los valores observados y las curvas de predicción desarrolladas a partir de las ecuaciones alométricas de aditividad para a) la biomasa de fuste (Bf), b) biomasa de ramas (Br), c) biomasa de ramillas con hojas (Brh) y d) biomasa total (Bt) de Pinus cembroides.

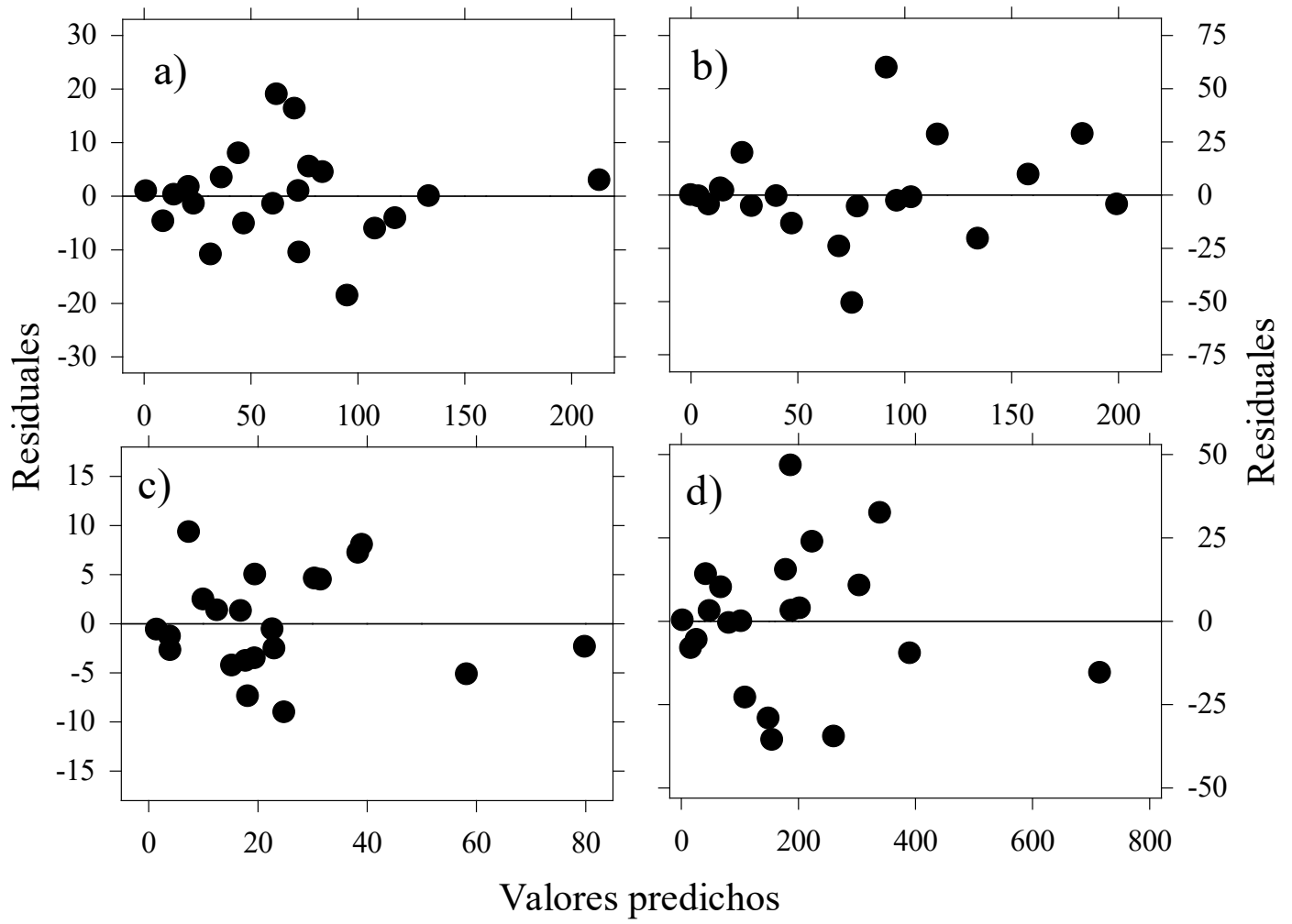

FIGURA 3. Valores predichos y residuales en la biomasa del a) fuste, b) ramas, c) ramillas con hojas y d) total en Pinus cembroides. 


\section{DISCUSIÓN}

Los dos conjuntos de ecuaciones de aditividad obtenidos en este estudio proporcionan una herramienta operacional para estimar con precisión la biomasa aérea de los árboles individuales de $P$. cembroides. La variación explicada por las ecuaciones de aditividad fue alta, esto se debe a que en su estructura se incluyeron como variables independientes al $D n$ y a la $H$. El uso de estas variables en la predicción de la biomasa ha sido discutido ampliamente por diferentes autores, debido a que pueden mejorar significativamente los resultados de ajuste y la capacidad de predicción (Bi et al., 2004; Li y Zhao, 2013), en lugar de emplear únicamente al $D n$ como variable predictora. Además, al incluir a la $H$ en las ecuaciones se tiene información relacionada sobre la competencia y/o fertilidad del sitio ocasionando estimaciones menos sesgadas (Tesfaye et al., 2015).

El uso del $D n$ como única variable predictora para la estimación de biomasa a nivel del árbol, ha mostrado buenos estadísticos de ajuste para diferentes especies de coníferas (Návar, González, Graciano, Dale y Parresol, 2004; Acosta-Mireles et al; 2002; Díaz-Franco et al., 2007). Sin embargo, la inclusión de la $H$ como segunda variable predictora tiene la ventaja de ser un factor predictivo que debe tomarse en cuenta en la cuantificación de la biomasa, debido a que se espera que en árboles con el mismo Dn, pero distinto ahusamiento, tengan alometrías diferentes (Nogueira, Fearnside, Nelson, Barbosa y Keizer, 2008; Temesgen et al., 2015). Además, la altura es importante para explicar las diferencias específicas en la arquitectura o la plasticidad fenotípica de los árboles dependiendo de las condiciones de crecimiento (Archibald y Bond, 2003). Una de las principales razones para omitir la altura del árbol es su dificultad para medirla en condiciones de dosel cerrado (Díaz-Franco et al., 2007; Hunter, Keller, Victoria y Morton, 2013; Larjavaara y Muller-Landau, 2013). No obstante, existen diferentes métodos que garantizan su correcta medición (Sullivan et al., 2018).

El sistema $\mathrm{S}_{2}$ basado en el modelo

$$
y=\beta_{0} \times D n^{\beta_{1}} \times H^{\beta_{2}}
$$

fue el que obtuvo mejores estadísticos de bondad de ajuste, expresando la relación entre la biomasa de los componentes con las variables dasométricas del árbol, por lo tanto, fue seleccionado para estimar la biomasa de los componentes específicos y del árbol individual de P. cembroides. Este modelo ha sido considerado como uno de los más flexibles ya que mejora la precisión de las estimaciones de la Bt y de los componentes de la copa, especialmente la biomasa de ramas y de las hojas (Zhou, Brandle, Schoeneberger y Awada, 2007; Dong, Zhang y Li, 2015).

Las ecuaciones de aditividad del $\mathrm{S}_{2}$ explican de $92 \%$ a $97 \%$ de la variabilidad de la biomasa de los componentes y 98\% de la variabilidad de la biomasa aérea total, valores que son diferentes a los señalados por Návar (2011) en modelos de potencia para estimar la partición de biomasa en $P$. cembroides, Pinus pinceana Gordon, Pinus pseudostrobus Brongn, Pinus brutia Ten y Cupressus arizonica Greene, incluyendo únicamente al $D n$ como variable predictora en especies de bosques templados secos del noreste de México, con coeficientes de determinación de 0.87, 0.60 y 0.81 para la biomasa del fuste, biomasa de hojas y ramas, y en la biomasa total, respectivamente. Estas diferencias pueden atribuirse a la alta variabilidad que se registra al integrar en un solo modelo de distribución de biomasa a cinco especies de coníferas, representadas por un número reducido de árboles por especie. Por el contrario, los resultados en este estudio son semejantes a los hallados por Chávez-Pascual, Rodríguez-Ortiz, Enríquez-del Valle, Velasco-Velasco y Gómez-Cárdenas (2017) en Pinus oaxacana Mirov bajo manejo en Ixtlán Oaxaca, México, quienes ajustaron modelos de la variable combinada generalizada para estimar la biomasa del fuste con corteza, biomasa de ramas y biomasa de hojas con coeficientes de determinación de $0.97,0.93$ y 0.98 , respectivamente; y un valor de 0.97 para el $\mathrm{R}^{2}$ adj en la ecuación de la biomasa total. Así mismo, a los obtenidos por Vargas-Larreta et al. (2017) quienes utilizaron ecuaciones de aditividad con la misma estructura a los de este estudio, con coeficientes de determinación de 0.85 a 0.95 para la biomasa área total y coeficientes de 0.63 a 0.97 en la distribución de biomasa en 11 especies de coníferas del noroeste de México. 
La aditividad o la compatibilidad es una característica deseable al desarrollar un sistema de ecuaciones de biomasa para eliminar la inconsistencia entre la suma de los valores predichos para los diferentes componentes (fuste, ramas y follaje) y la predicción total del árbol (Bi et al., 2004; Dong et al., 2015), propiedad que frecuentemente es ignorada. En México son limitados los estudios que toman en cuenta estas relaciones de aditividad, por ejemplo, Návar et al. (2004), desarrollaron ecuaciones aditivas ponderadas para el cálculo de la biomasa en plantaciones de Pinus durangensis Martínez, Pinus cooperi Blanco y Pinus engelmannii Carr. de la Sierra Madre Occidental en Durango, México, de igual manera, Monroy-Rivera y Návar-Cháidez (2004) generaron ecuaciones de aditividad para componentes de biomasa en plantaciones comerciales de Hevea brasiliensis en Veracruz, México y Aquino-Ramírez et al. (2015) propusieron un sistema de ecuaciones de partición de biomasa con aditividad para tres especies de árboles tropicales en Pluma Hidalgo Oaxaca, México. Recientemente, Vargas-Larreta et al. (2017) ajustaron simultáneamente sistemas de ecuaciones para la biomasa de los componentes y la biomasa total para asegurar la aditividad, en 17 especies forestales en los bosques templados del noroeste de México, así mismo, Flores-Medina et al. (2018) desarrollaron ecuaciones alométricas considerando la aditividad para estimar biomasa por fracción de grosor y total en cuatro especies en etapa de regeneración del suroeste de Durango, México.

La proporción de biomasa en las ramas se incrementó conforme aumentaron los tamaños del árbol. En las categorías diamétricas de $25 \mathrm{~cm}$ a $40 \mathrm{~cm}$ la asignación de biomasa superó más de $40 \%$ a la biomasa del fuste (Fig. 1). Estos resultados son consistentes con el estudio realizado por Grier, Elliott y McCullough, (1992) en un bosque mixto de Piñón-Juniperus en el norte de Arizona, encontraron que la asignación de biomasa en las ramas de Pinus edulis Engelm fue de $43.7 \%$ en rodales jóvenes con $D n$ promedio de 16 $\mathrm{cm}$ y se incrementó a $51.1 \%$ en rodales maduros con $D n$ promedio de $25 \mathrm{~cm}$. Los patrones de asignación de biomasa en los pinos piñoneros son diferentes a la mayoría de las especies del género Pinus donde la mayor cantidad de biomasa se concentra principalmente en el fuste (ChávezAguilar et al., 2016; Vargas-Larreta et al., 2017). Lo anterior se atribuye a que los árboles que crecen en espacios abiertos tienden a presentar copas extendidas (Návar, 2009), porque las ramas no tienen una buena poda natural lo que favorece un crecimiento libre y, en consecuencia, el árbol destina una mayor proporción de biomasa a los tejidos relacionados con el soporte del follaje y a las estructuras reproductivas (Loveall y Harrington, 2008).

Las ecuaciones de biomasa con aditividad presentadas en este estudio aportarán información más eficiente para mejorar la cuantificación de la biomasa y carbono, con el propósito de ayudar en el manejo sustentable de los bosques piñoneros de $P$. cembroides. Se recomienda la aplicación de las ecuaciones dentro del intervalo de diámetros en el que fueron construidas porque las relaciones alométricas no son constantes y pueden cambiar de diferentes maneras según la especie, el tamaño, calidad del sitio y la edad (Riofrío et al., 2015), lo que ocasionaría resultados con menor precisión.

\section{CONCLUSIONES}

El sistema de ecuaciones alométricas $\left(\mathrm{S}_{2}\right)$ propuesto que integró al diámetro normal y la altura total del árbol como variables predictoras permite estimaciones robustas de la biomasa de los componentes estructurales y la biomasa aérea total de Pinus cembroides, asegurando la aditividad de los componentes.

En los árboles de $P$. cembroides la proporción de biomasa por componentes fue de $43.2 \%, 41.2 \%$ y $15.5 \%$ para fuste, ramas y ramillas con hojas, sin embargo, en las categorías diamétricas de $25 \mathrm{~cm}$ a $40 \mathrm{~cm}$ la acumulación de biomasa fue mayor en ramas que la del fuste con más de $40 \%$.

Las ecuaciones desarrolladas en este estudio pueden aplicarse de manera sencilla y precisa a datos de árboles individuales e inventarios forestales para estimaciones de biomasa y carbono, lo que contribuirá al desarrollo de prácticas de manejo sustentable y estrategias de conservación de la especie, donde la producción de madera no es la función principal. 


\section{RECONOCIMIENTOS}

Los autores expresan su agradecimiento a las autoridades de los Bienes Comunales de Sombrerete, municipio de Cadereyta, Querétaro, México por las facilidades y apoyo recibido para la realización de este estudio.

\section{REFERENCIAS}

Acosta-Mireles, M., Vargas-Hernández, J., Velázquez-Martínez, M., \& Etchevers-Barra, J. D. (2002). Estimación de la biomasa aérea mediante el uso de relaciones alométricas en seis especies arbóreas en Oaxaca, México. Agrociencia, 36, 725-736.

Aquino-Ramírez, M., Velázquez-Martínez, A., Castellanos-Bolaños, J. F., De los Santos-Posadas, H., \& Etchevers-Barra, J. D. (2015). Partición de la biomasa aérea en tres especies arbóreas tropicales. Agrociencia, 49(3), 299-314.

Archibald, S. \& Bond, W.J., 2003. Growing tall vs growing wide: tree architecture and allometry of Acacia karroo in forest, savanna, and arid environments. Oikos, 102(1), 3-14. doi: 10.1034/j.16000706.2003.12181.x

Bi, H., Murphy, S., Volkova, L., Weston, C., Fairman, T., Li, Y., Law, R., Norris, J., Lei, X., \& Caccamo, G. (2015). Additive biomass equations based on complete weighing of sample trees for open eucalypt forest species in south-eastern Australia. Forest Ecology Management, 349,106-121. doi: 10.1016/j.foreco.2015.03.007

Bi, H., Turner, J., \& Lambert, M. J. (2004). Additive biomass equations for native eucalypt forest trees of temperate Australia. Trees, 18(4), 467-479. doi: 10.1007/s00468-004-0333-z

Carrillo-Anzures, F., Acosta-Mireles, M., Jiménez-Cruz, C. D. R., González-Molina, L., \& Etchevers-Barra, J. D. (2016). Ecuaciones alométricas para estimar la biomasa y el carbono de la parte aérea de Pinus hartwegii en el Parque Nacional Ixta-Popo, México. Revista Mexicana de Ciencias Agricolas, 7(3), 681-691. doi: 10.29312/remexca.v7i3.327

Carvalho, J. P. \& Parresol, B. R. (2003). Additivity in tree biomass components of Pyrenean Oak (Quercus pyrenaica Willd.). Forest Ecology Management, 179, 269-276. doi: 10.1016/S03781127(02)00549-2

Constante-García, V., Villanueva-Díaz, J., Cenaro-Paredes, J., CornejoOviedo, E. H., \& Valencia-Manzo, S. (2009). Dendrocronología de Pinus cembroides Zucc y reconstrucción de precipitación estacional para el sureste de Coahuila. Revista Ciencia Forestal en México, 34(106), 17-39.

Chave, J., Andalo, C., Brown, S., Cairns, M.A., Chambers, J.Q., Eamus, D., Fölster, H., Fromard, F., Higuchi, N., Kira, T., Lescure, J. P.,
Nelson, B.W., Ogawa, H., Puig, H., Riéra, B., \& Yamakura, T. (2005). Tree allometry and improved estimation of carbon stocks and balance in tropical forests. Oecologia, 145(1), 87-99. doi: 10.1007/s00442-005-0100-x

Chávez-Aguilar, G., Ángeles-Pérez, G., Pérez-Suárez, M., López-López, M. A., García-Moya, E., \& Wayson, C. (2016). Distribución de biomasa aérea en un bosque de Pinus patula bajo gestión forestal en Zacualtipán, Hidalgo, México. Madera y Bosques, 22(3), 23-36. doi: $10.21829 /$ myb.2016.2231454

Chávez-Pascual, E. Y., Rodríguez-Ortiz, G., Enríquez-del Valle, J. R., Velasco-Velasco, V. A. \& Gómez-Cárdenas, M. (2017). Compartimentos de biomasa aérea en rodales de Pinus oaxacana bajo tratamientos silvícolas. Madera y Bosques, 23(3), 147-161. doi: 10.21829/myb.2017.2331627

Daryaei, A. \& Sohrabi, H. (2016). Additive biomass equations for small diameter trees of temperate mixed deciduous forests. Scandinavian Journal of Forest Research, 31(4), 394-398. doi: 10.1080/02827581.2015.1089932

Díaz-Franco, R., Acosta-Mireles, M., Carrillo-Anzures, F., BuendíaRodríguez, E., Flores-Ayala, E., \& Etchevers-Barra, J. D. (2007). Determinación de ecuaciones alométricas para estimar biomasa y carbono en Pinus patula Schl. et Cham. Madera y Bosques, 13(1), 2534. doi: 10.21829/myb.2007.1311233

Dong, L., Zhang, L., \& Li, F. (2015). Developing additive systems of biomass equations for nine hardwood species in Northeast China. Trees, 29(4), 1149-1163. doi: 10.1007/s00468-015-1196-1

Dong, L., Zhang, L., Li, F. (2014). A compatible system of biomass equations for three conifer species in Northeast, China. Forest Ecology and Management, 329, 306-317. doi: 10.1016/j.foreco.2014.05.050

Douterlungne, D., Herrera-Gorocica, A. M., Ferguson, B. G., Siddique, I., \& Soto-Pinto, L. (2013). Ecuaciones alométricas para estimar biomasa y carbono de cuatro especies leñosas neotropicales con potencial para la restauración. Agrociencia, 47(4), 385-397.

Eker, M., Poudel, K. P., \& Özçelik, R. (2017). Aboveground biomass equations for small trees of brutian pine in Turkey to facilitate harvesting and management. Forests, 8(12), $477 . \quad$ doi: 10.3390/f8120477

Flores-Medina, F., Vega-Nieva, D., Corral-Rivas, J., Álvarez-González, J., Ruiz-González, A., López-Sánchez, C., \& Carillo Parra, A. (2018). Desarrollo de ecuaciones alométricas de biomasa para la regeneración de cuatro especies en Durango, México. Revista Mexicana de Ciencias Forestales, 9(46). doi: 10.29298/rmcf.v9i46.119

García, E. (2004). Modificaciones al sistema de clasificación climática de Koppen (5a ed.). México: UNAM. 
Greene, W. H. (1999). Econometric Analysis (4a ed.). Upper Saddle River, N.J.: Prentice Hall.

Grier, C. C., Elliott, J. K., \& McCullough, G. D. (1992). Biomass distribution and productivity of Pinus edulis-Juniperus monosperma woodlands of north-central Arizona. Forest Ecology and Management, 50(1992), 331-350. Recuperado de https://www.srs.fs.usda.gov/pubs/ja/ja_grier001.pdf

Hunter, M. O., Keller, M., Victoria, D., \& Morton, D. C. (2013). Tree height and tropical forest biomass estimation. Biogeosciences, 10(12), 8385-8399. doi: 10.5194/bg-108385-2013

Larjavaara, M., \& Muller-Landau, H. C. (2013). Measuring tree height: a quantitative comparison of two common field methods in a moist tropical forest. Methods in Ecology and Evolution,4(9), 793-801. doi: 10.1111/2041-210X.12071

Li, H., \& Zhao, P. (2013). Improving the accuracy of tree-level aboveground biomass equations with height classification at a large regional scale. Forest Ecology and Management, 289, 153-163. doi: $10.1016 /$ j.foreco.2012.10.002

Lin, K., Lyu, M., Jiang, M., Chen, Y., Li, Y., Chen, G., Xie, J., \& Yang, Y. (2017). Improved allometric equations for estimating biomass of the three Castanopsis carlesii $H$. forest types in subtropical China. New Forest, 48(1), 115-135. doi: 10.1007/s11056-016$9559-z$

Loveall, M. \& Harrington, J. T. (2008). Description and prediction of individual tree biomass on pinon (Pinus edulis) in northern New Mexico. In G. J. Gottfried, J. D. Shaw, \& P. L. Ford (Comps.), Ecology, management, and restoration of piñon juniper and ponderosa pine ecosystems: Combined proceedings of the 2005 St. George, Utah and 2006 Albuquerque, New Mexico workshops (pp. 28-34) (Proc. Pap. RMRSP-51). Rocky Mountain Research Station, Fort Collins, CO: USDA For. Serv. Recuperado de https://www.fs.fed.us/rm/pubs/rmrs_p051/rmrs_p051_028_0 34.pdf

Luna-Cavazos, M., Romero-Manzanares, A., \& García-Moya, E. (2008). Afinidades en la flora genérica de piñonares del norte y centro de México: un análisis fenético. Revista Mexicana de Biodiversidad, 79(2), 449-458.

Meng, S., Liu Q., Zhou, G., Jia. Q., Zhuang, H., \& Zhou, H. (2017). Aboveground tree additive biomass equations for two dominant deciduous tree species in Daxing'anling, northernmost China. Journal of Forest Research, 22(4), 233-240. doi: 10.1080/13416979.2017.1333277

Mensah, S., Veldtman, R., \& Seifert, T. (2017): Allometric models for height and aboveground biomass of dominant tree species in
South African Mistbelt forests. Southern Forests: a Journal of Forest Science, 79(1), 19-30. doi: 10.2989/20702620.2016.1225187

Monroy-Rivera, C., \& Návar-Cháidez, J. de J. (2004). Ecuaciones de aditividad para estimar componentes de biomasa de Hevea brasiliensis Muell. Arg. en Veracruz, México. Madera y Bosques, 10(2), 29-43. doi: 10.21829/myb.2004.1021273

Návar, J. (2011). Plasticity of biomass component allocation patterns in semiarid Tamaulipan thornscrub and dry temperate pine species of northeastern Mexico. Polibotanica, 31, 121-141. Recuperado de http://polibotanica.mx/pages/en/index/31-40/num.31.php?lang $=\mathrm{EN}$

Návar, C. J. J., González, B. N., Graciano, L. J. J., Dale, V., \& Parresol, B. (2004). Additive biomass equations for pine species of forest plantations of Durango, Mexico. Madera y Bosques, 10(2), 17-28. doi: $10.21829 /$ myb.2004.1021272

Nogueira, E. M., Fearnside, P. M., Nelson, B. W., Barbosa, R. I., \& Keizer, E. W. H. (2008). Estimates of forest biomass in the Brazilian Amazon: new allometric equations and adjustments to biomass from wood-volume inventories. Forest Ecology and Management, 256(11), 1853-1867. doi: 10.1016/j.foreco.2008.07.022

Parresol, B. R. (2001). Additivity of nonlinear biomass equations. Canadian Journal Forest Research, 31(5), 865-878. doi: 10.1139/x00202

Picard, N, Saint-Andre L., \& Henry, M. (2012). Manual for building tree volume and biomass allometric equations from field measurement to prediction. Montpellier: Food and Agricultural Organization of the United Nations, Rome, and Centre Coopération Internationale en Recherche Agronomique pour le Développement,

Pompa-García, M. \& Yerena-Yamalliel, J. I. (2014). Concentración de carbono en Pinus cembroides Zucc: Fuente potencial de mitigación del calentamiento global. Revista Chapingo Serie Ciencias Forestales y del Ambiente, 20(3), 169-175. doi: 10.5154/r.rchscfa.2014.04.014

Quiñonez-Barraza, G., Zhao, D., de los Santos-Posadas, H. M., Santiago-García, W., Tamarit-Urias, J. C., \& Nájera-Luna, J. A. (2019). Sistema compatible de ahusamiento, volumen, peso verde, biomasa y concentración de carbono para Quercus sideroxyla Bonpl. Revista Chapingo Serie Ciencias Forestales y del Ambiente, 25(1), 49-69. doi: 10.5154/r.rchscfa.2018.06.050

Riofrío, J., Herrero, C., Grijalva, J., \& Bravo, F. (2015). Aboveground tree additive biomass models in Ecuadorian highland agroforestry systems. Biomass and Bioenergy, 80, 252-259. doi: 10.1016/j.biombioe.2015.05.026 
Ríos C., E. de los, De Hoogh, R., \& Návar C., J. J. (2008). Ensayo de especies con pinos piñoneros en el nordeste de México. Revista Chapingo Serie Ciencias Forestales y del Ambiente, 14(2), 97-104. Recuperado

\section{de} https://chapingo.mx/revistas/forestales/contenido.php?section $=$ article\&id_articulo $=496 \&$ doi $=0000$

Rojas-García, F., De Jong, B. H., Martínez-Zurimendí, P., \& Paz-Pellat, F. (2015). Database of 478 allometric equations to estimate biomass for Mexican trees and forests. Annals of forest science, 72(6), 835-864. doi: 10.1007/s13595-015-0456-y

Rosas-Chavoya, M., Granados-Sánchez, D., Granados-Victorino, R. L., \& Esparza-Govea, S. (2016). Clasificación y ordenación de bosques de pino piñonero del estado de Querétaro. Revista Mexicana de Ciencias Forestales, 7(33), 52-73. doi: 10.29298/rmcf.v7i33.90

SAS Institute Inc. 2004. SAS/ETS® 9.1 User's Guide. Cary, NC. 2416 p.

Skovsgaard, J. P. \& Vanclay, J. K. (2013). Forest site productivity: a review of spatial and temporal variability in natural site conditions. Forestry, 86(3), 305-315. doi: 10.1093/forestry/cpt010

Sullivan, M. J., Lewis, S. L., Hubau, W., Qie, L., Baker, T. R., Banin, L. F., ..., \& Phillips, O. L. (2018). Field methods for sampling tree height for tropical forest biomass estimation. Methods in ecology and evolution, 9(5), 1179-1189. doi: 10.1111/2041-210X.12962

Temesgen, H., Affleck, D., Poudel, K., Gray, A., \& Sessions, J. (2015). A review of the challenges and opportunities in estimating above ground forest biomass using tree-level models. Scandinavian Journal of Forest Research, 30(4), 326-335. doi: 10.1080/02827581.2015.1012114

Tesfaye, M. A., Bravo-Oviedo, A., Bravo, F., \& Ruiz-Peinado, R. (2015). Aboveground biomass equations for sustainable production of fuelwood in a native dry tropical afro-montane forest of Ethiopia. Annals of Forest Science, 73(2), 411-423. doi: 10.1007/s13595-0150533

Vargas-Larreta, B., López-Sánchez, C. A., Corral-Rivas, J. J., LópezMartínez, J. O., Aguirre-Calderón, C. G., \& Álvarez-González, J.
G. (2017). Allometric equations for estimating biomass and carbon stocks in the temperate forests of North-Western Mexico. Forests, 8 (8), 269. doi: 10.3390/f8080269

Wang, X., Bi, H., Ximenes, F., Ramos, J., \& Li, Y. (2017). Product and residue biomass equations for individual trees in rotation age Pinus radiata stands under three thinning regimes in New South Wales, Australia. Forests, 8(11), 439. doi: 10.3390/f8110439

Zhang, Q., Wang, C., Wang, X., \& Quan, X. (2009). Carbon concentration variability of 10 Chinese temperate tree species. Forest Ecology Management, 258(5), 722-727. doi: 10.1016/j.foreco.2009.05.009

Zhao, D. H., Kane, M., Markewitz, D., Teskey, R., \& Clutter, M. (2015). Additive tree biomass equations for Midrotation loblolly pine plantations. Forest Science, 61(4), 613-623. doi: 10.5849/forsci.14193

Zhou, X, Brandle, J. R., Schoeneberger, M. M., \& Awada, T. (2007). Developing above-ground woody biomass equations for opengrown, multiple-stemmed tree species: shelterbelt-grown Russian-olive. Ecological Modelling, 202(3-4), 311-323. doi: 10.1016/j.ecolmodel.2006.10.024

Manuscrito recibido el 12 de julio de 2018

Aceptado el 12 de abril de 2019

Publicado el 17 de febrero de 2020

Este documento se debe citar como:

Cuevas C., J. C. \& Aquino R., M. (2020). Ecuaciones de aditividad para la estimación de biomasa aérea de Pinus cembroides Zucc. Madera y Bosques, 26(1), e2611821. doi: 10.21829/myb.2020.2611821

Madera y Bosques por Instituto de Ecología, A.C. se distribuye bajo una Licencia Creative Commons Atribución-NoComercialCompartirlgual 4.0 Internacional. 